\title{
Enhancing Drug Overdose Alerts with Spatial Visualization
}

\section{Robert Gottlieb, Kayley Dotson, Amanda Billman}

Indiana State Department of Health, Indianapolis, Indiana, United States

Objective

This poster presentation shares Indiana's approach of alerting local health departments (LHDs) with near real-time drug overdose data and how this process has been enhanced through mapping and analysis with a geographic information system (GIS).

\section{Introduction}

Since 2008, drug overdose deaths exceeded the number of motor vehicle traffic-related deaths in Indiana, and the gap continues to widen [1]. While federal funding opportunities are available for states, it often takes years for best practices to be developed, shared, and published. Similarly, local health departments (LHDs) may experience lengthy delays to receive finalized county health statistics.

Indiana collects and stores syndromic emergency department data in the Public Health Emergency Surveillance System (PHESS) and uses the Electronic Surveillance System for the Early Notification of Community-based Epidemics version 1.21 (ESSENCE) to monitor public health events and trends. In July 2017, the Indiana Overdose Surveillance Team (IOST) developed a standard process for monitoring and alerting local health partners of increases in drug overdoses captured in ESSENCE at the county level. ISDH is enhancing these alerts by mapping the data in GIS and providing spatiotemporal data to LHDs to inform more targeted intervention and prevention efforts.

\section{Methods}

The IOST monitors drug overdoses statewide by analyzing daily queries from ESSENCE and sending email alerts to LHDs that are experiencing a statistically significant increase in suspected overdose activity at a hospital or county level. The IOST then requests that LHDs complete an overdose response feedback survey describing their actions after receiving an overdose alert.

The IOST GIS analyst has enhanced overdose alerts by utilizing daily emergency department data queries from the PHESS database based on chief complaint and diagnosis text. Python ${ }^{\mathrm{TM}}$ and ArcGIS ${ }^{\mathrm{TM}}$ are used to deduplicate and geocode records, calculate the rate of cases within a hexagonal grid, and calculate the kernel density of case counts to show patterns at the neighborhood level. Comparisons to previous time periods are also calculated. Temporal and spatial scales of analysis are flexible, but 7 days and 30 days are used most often. Results are mapped in an HTML file using an open source Python package for dissemination to LHDs.

\section{Results}

Between July 26, 2017, and Sept. 4, 2018, the IOST sent 89 suspected overdose alerts to LHDs. Alerts were sent to 45 different LHDs, of which 22 received multiple alerts (range: 1-9 repeat alerts). LHDs were requested to complete the survey on their initial alert, and a total of 31 jurisdictions completed this survey $(31 / 45=69 \%)$. The majority of the LHD respondents $(27 / 31=87 \%)$ wanted to continue receiving overdose alert emails.

Our enhanced spatial analysis project has mapped more than 500 cases per week. Geocoding was successful for approximately $87 \%$ of the addresses received through PHESS. Neighborhoods in urban areas with higher counts have been identified, though variability from week to week is high. Areas of high overdose rates that cross county boundaries have also been detected, which would not have been possible using ESSENCE alone.

\section{Conclusions}

Notifying LHDs of near real-time drug overdose trends is a catalyst for drug overdose planning and response efforts in Indiana. GIS mapping of the data provides an easy way for LHDs to view and share spatial trends with their local planning partners and identify community intervention strategies that can reduce drug overdose rates and improve outcomes for overdose survivors.

SDS Annual Conference Proceedings 2019. This is an Open Access article distributed under the terms of the Creative Commons AttributionNoncommercial 4.0 Unported License (http://creativecommons.org/licenses/by-nc/3.0/), permitting all non-commercial use, distribution, and reproduction in any medium, provided the original work is properly cited. 


\section{Acknowledgement}

Chris Waldron, Garry Raynor, Peter Krombach, Harold Gil

\section{References}

1. Overdose Prevention [Internet]. Indianapolis: Indiana State Department of Health; 2017. Indiana Special Emphasis Report: Drug Overdose Deaths 1999-2015; August 2017. [cited 2017 Sept 25]. Available from: http://www.in.gov/isdh/files/2017_SER_Drug_Deaths_Indiana.pdf

\section{Figure 1}

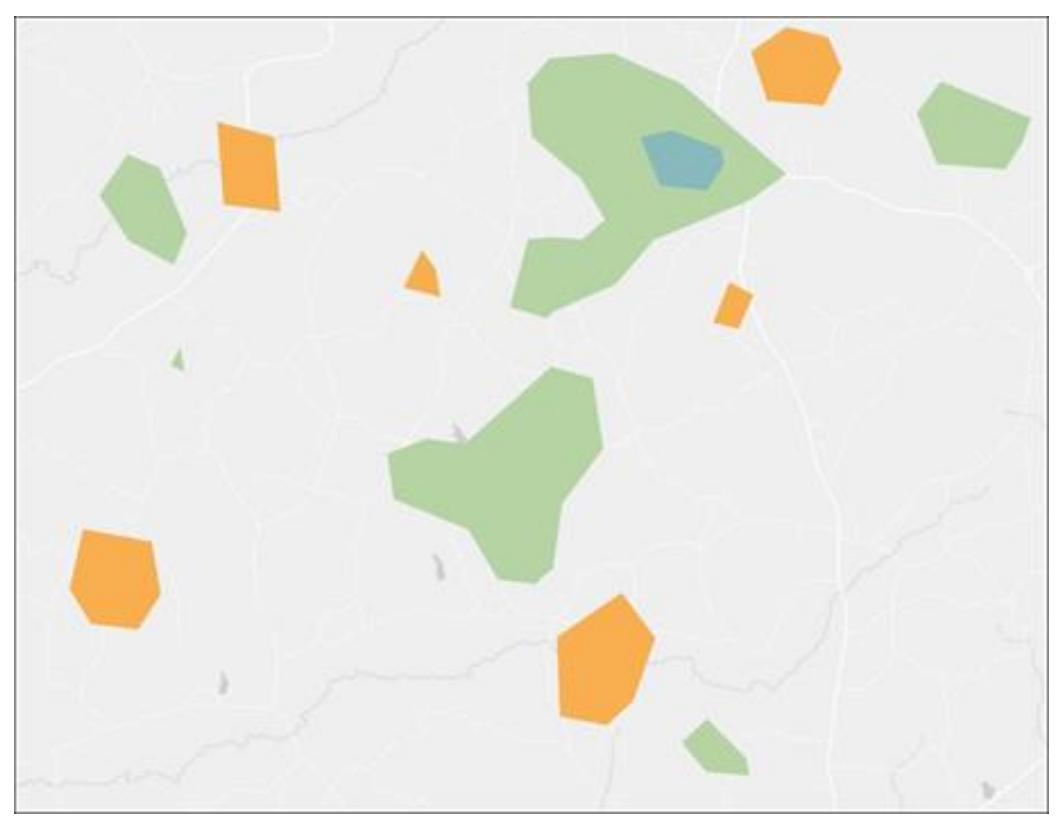

ISDS Annual Conference Proceedings 2019. This is an Open Access article distributed under the terms of the Creative Commons AttributionNoncommercial 4.0 Unported License (http://creativecommons.org/licenses/by-nc/3.0/), permitting all non-commercial use, distribution, and reproduction in any medium, provided the original work is properly cited. 


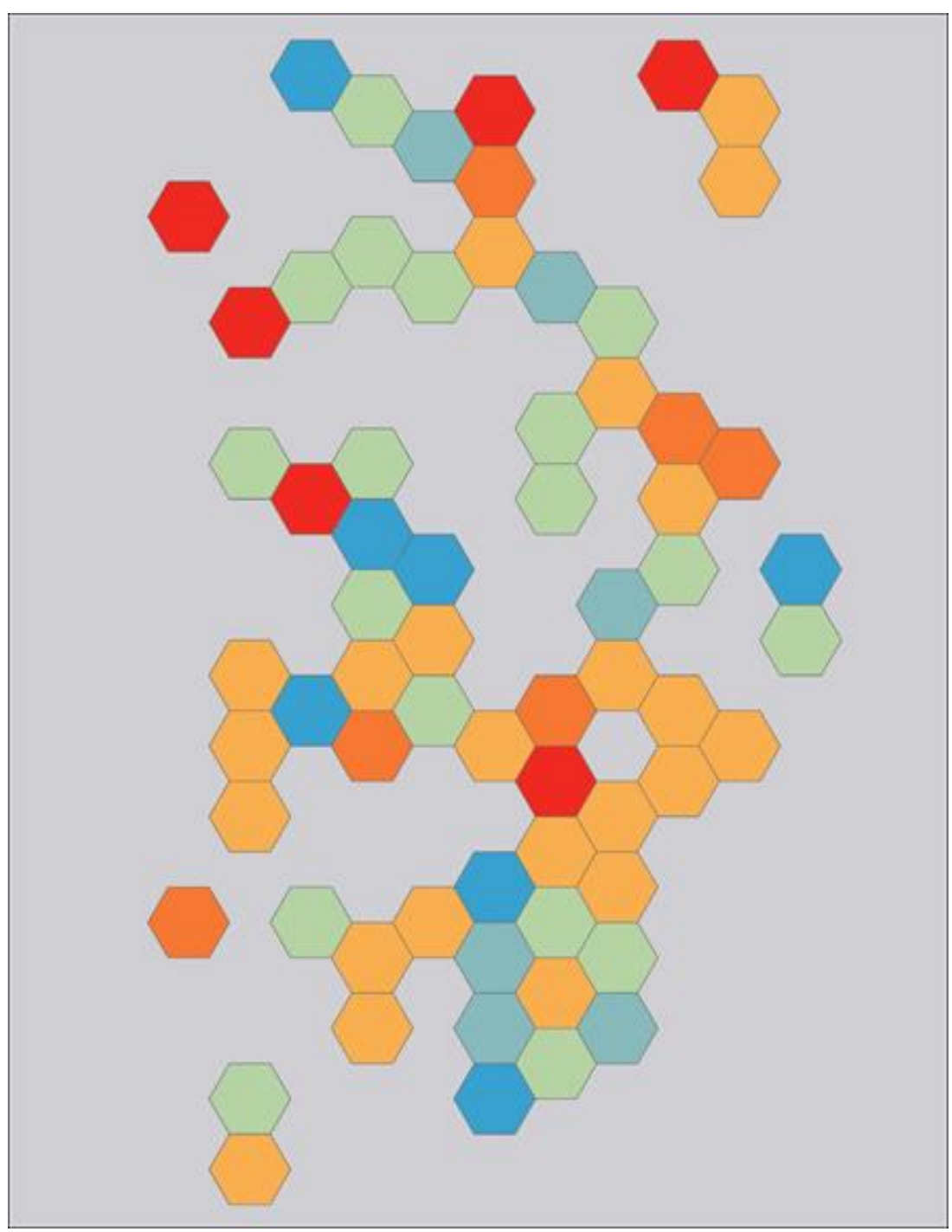

Figure 2 